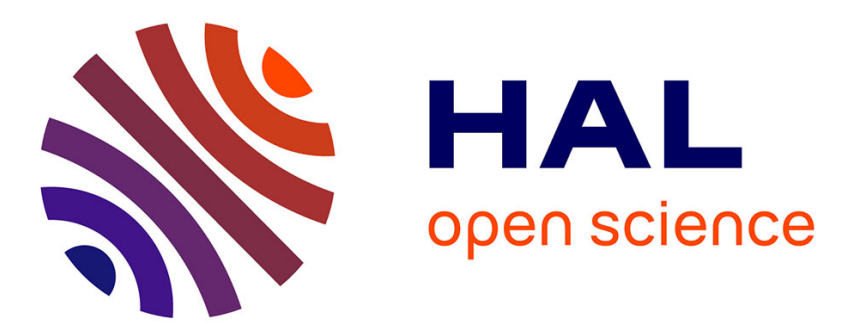

\title{
Determination of the optical properties and size dispersion of Si nanoparticles within a dielectric matrix by spectroscopic ellipsometry
}

\author{
A. -S. Keita, A. En Naciri, Y. Battie, F. Delachat, Marzia Carrada, G. \\ Ferblantier, A. Slaoui
}

\section{To cite this version:}

A. -S. Keita, A. En Naciri, Y. Battie, F. Delachat, Marzia Carrada, et al.. Determination of the optical properties and size dispersion of Si nanoparticles within a dielectric matrix by spectroscopic ellipsometry. Journal of Applied Physics, 2014, 116 (10), pp.103520. 10.1063/1.4894619 . hal01517452

\section{HAL Id: hal-01517452 \\ https://hal.univ-lorraine.fr/hal-01517452}

Submitted on 11 May 2017

HAL is a multi-disciplinary open access archive for the deposit and dissemination of scientific research documents, whether they are published or not. The documents may come from teaching and research institutions in France or abroad, or from public or private research centers.
L'archive ouverte pluridisciplinaire HAL, est destinée au dépôt et à la diffusion de documents scientifiques de niveau recherche, publiés ou non, émanant des établissements d'enseignement et de recherche français ou étrangers, des laboratoires publics ou privés. 
Determination of the optical properties and size dispersion of Si nanoparticles within a dielectric matrix by spectroscopic ellipsometry
A.-S. Keita', A. En Naciri', Y. Battie, F. Delachat, M. Carrada, G. Ferblantier, and A. Slaoui

Citation: Journal of Applied Physics 116, 103520 (2014); doi: 10.1063/1.4894619

View online: http://dx.doi.org/10.1063/1.4894619

View Table of Contents: http://aip.scitation.org/toc/jap/116/10

Published by the American Institute of Physics

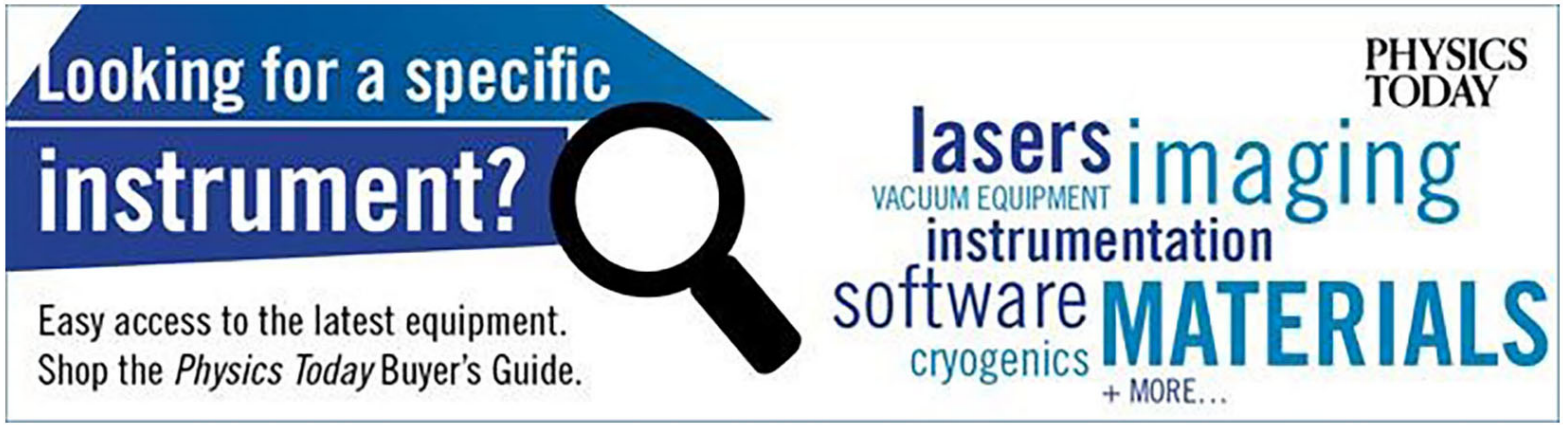




\title{
Determination of the optical properties and size dispersion of $\mathrm{Si}$ nanoparticles within a dielectric matrix by spectroscopic ellipsometry
}

\author{
A.-S. Keita, ${ }^{1,2, a)}$ A. En Naciri, ${ }^{1, b)}$ Y. Battie, ${ }^{1}$ F. Delachat, ${ }^{3,4}$ M. Carrada, ${ }^{3,5}$ G. Ferblantier, ${ }^{3}$ \\ and A. Slaoui ${ }^{3}$ \\ ${ }^{1}$ LCP-A2MC, Institut Jean Barriol, Université de Lorraine, 1 Bd Arago, 57070 Metz, France \\ ${ }^{2}$ Max Planck Institute for Intelligent Systems (formerly Max Planck Institute for Metals Research), \\ Heisenbergstraße 3, D-70569 Stuttgart, Germany \\ ${ }^{3}$ ICube, Université de Strasbourg-CNRS, 23 rue du Loess BP20, 67037 Strasbourg Cedex 2, France \\ ${ }^{4}$ CEA-Leti, 17 rue des Martyrs, 38054 Grenoble, France and INRS Centre Energie, Matériaux et \\ Télécommunications, 800 de la Gauchetière Ouest, Montréal, Québec H5A 1K6, Canada \\ ${ }^{5}$ CEMES-CNRS, nMat Group, 29 rue J. Marvig, 31055 Toulouse, France
}

(Received 11 June 2014; accepted 23 August 2014; published online 12 September 2014)

\begin{abstract}
We report on a comparative study between dielectric functions of $\mathrm{Si}$ nanoparticles (Si-NPS) obtained from Bruggeman effective medium approximation (BEMA), Maxwell-Garnett (MG), and a modified Maxwell-Garnett $(M M G)$ models. Unlike $B E M A$ and $M G$, a size-distribution dependent dielectric function of $\mathrm{Si}-\mathrm{NPs}$ is considered in the introduced $M M G$ model. We show that the standard deviation $\sigma$ of a size distribution can be evaluated by analyzing the imaginary part of the dielectric functions of Si-NPs extracted from BEMA and $M M G$. In order to demonstrate this, several samples composed of $\mathrm{Si}$-NPs embedded in silicon-rich silicon nitride are investigated by spectroscopic ellipsometry over the photon energy range varying between 2 and $4 \mathrm{eV}$. Assuming a lognormal size distribution of the Si nanoparticles, it is evidenced that the parameter $\sigma$ ranges between 1.15 and 1.35. The values of size dispersion deduced by this methodology are in good agreement with TEM observations. C 2014 AIP Publishing LLC. [http://dx.doi.org/10.1063/1.4894619]
\end{abstract}

\section{INTRODUCTION}

A precise evaluation and control of the size distribution of nanomaterials has been a task of primarily interest for building up nanostructured components with reliable and optimal (mainly optical and electrical) properties, knowing that the reproducible preparation of rigorously monodisperse nanocomposites is rather hard to achieve. ${ }^{1,2}$ For example, the gap energy and the photoluminescence efficiency of semiconductor nanocrystals are significantly changed when their size dispersion gets broader. ${ }^{3,4}$ In order to achieve this task, structural characterization techniques may be employed such as transmission electron microscopy (TEM). This technique is widely used in order to get the size distribution of the nanostructures; nonetheless, it allows probing only locally their structural properties. ${ }^{5,6}$

Alternatively the use of optical techniques, for the accomplishment of this task, may be possible but remains somehow quite challenging, since size distribution is most of the time deduced indirectly through some specific physical effects such as electron-photon ${ }^{4,7}$ and electron-phonon ${ }^{8-10}$ interactions. Thus, in Raman spectroscopy, the size dependence can be observed through the broadening and the line shift (asymmetry) of the Raman peak. However, the widening of a Raman peak could also be induced by stress and therefore a clear demarcation between effects originating from size and stress, respectively, may not be conspicuous., ${ }^{9,11,12}$

By employing absorption spectroscopy, Pesika et al. showed that, in the dilute concentration limit, the size

\footnotetext{
a)a.keita@is.mpg.de

b) aotmane.en-naciri@univ-lorraine.fr
}

distribution $n(R)$ of $\mathrm{ZnO}$ quantum particles can be related to the local slope of their absorbance spectrum $d A / d R$ by an expression of the type ${ }^{13}$

$$
n(R) \propto-\frac{d A / d R}{4 \pi R^{3} / 3} .
$$

Combining $U V$ spectroscopy and TEM characterization, these authors demonstrated that the particle size distribution can be evaluated from the analysis of the absorption edge. ${ }^{13}$ Hence, the size distribution may have appreciable effects on the dielectric function of semiconductor nanoparticles of relatively large size.

In the two well-known effective medium approximations-Maxwell-Garnett $(M G)$ and Bruggeman effective medium approximation (BEMA) - that have been mostly employed so far for the derivation of the optical properties of silicon nanoparticles, the microtopology of the composite film is described by the volume fraction only. Bergmann has shown that the dielectric function of a twophase composite is connected with the microstructure of the composite medium $^{14}$

$$
\varepsilon_{e f f}=\varepsilon_{2} \cdot\left(1-f \int_{0}^{1} \frac{G(L)}{t-L} d L\right)
$$

where $f, \varepsilon_{1}, \varepsilon_{2}$, and $\varepsilon_{e f f}$ are the volume fraction and dielectric function of the component 1, 2, and effective medium, respectively; $G(L)$ is the spectral function; and $t$, defined as: $t=\frac{\varepsilon_{2}}{\varepsilon_{2}-\varepsilon_{1}}$, can be connected to the dielectric contrast between the matrix and the nanoparticles. The spectral function is a normalized distribution function which brings information 
about the geometry. The equations describing $M G$ and BEMA models obey to the Bergman theorem. ${ }^{15}$

The application of such effective medium theories should be questionable at the nanometer scale in various (strong, intermediate, weak) confinement regimes. ${ }^{16}$ Indeed, they do not show any dependence of the dielectric function with the size distribution of the nanoparticles. Nonetheless, the polarizability of a nanomaterial depends explicitly on its size and is related to its macroscopic dielectric constant as recalled in Ref. 17. It has also to be noted that at the nanometer scale, the quasistatic approximation $2 \pi R \ll \lambda$ is fulfilled, so that the dielectric function represents an average quantity of the response of the ensemble of (nano) inclusions. ${ }^{18}$ Thus in $M G$ and BEMA, the correlation between the optical properties and average size of an ensemble of nanoparticles is made through their volume fraction $f$.

$B E M A$ and $M G$ represent mean field models in which the effective dielectric function is independent of the dispersion $\sigma$. Therefore, such models will correctly describe a composite medium only in the case of narrow $(M G)$ or broad (BEMA) size distributions. ${ }^{19}$ Previously, the insertion of size ${ }^{20,21}$ distribution in an effective medium model such as $M G$ has been carried out, particularly in order to explain the strong infrared absorption of metal nanoparticles. Besides in the classical $B E M A$, it is not possible to deduce any information regarding the size distribution of the (nano) particles since the two components are in close proportions and form an aggregated microstructure. Few extensions of BEMA have been set forward in order to take into account the influence of size distribution by considering the contribution of higher order multipoles. ${ }^{22}$

The accurate and reliable determination of the dielectric function of semiconductor nanoparticles by spectroscopic ellipsometry has remained a problematic issue. We have recently shown that the striking discrepancies observed in the literature, for the dielectric functions of Si-NPs, could partly come from the fact that size distribution was not taken into account in the modeling of the ellipsometric data. ${ }^{23,24}$ Going further in this paper, we demonstrate that a comparison between the optical properties calculated by BEMA and $M G$ can provide quantitative information about the size dispersion $\sigma$ of semiconductor nanoparticles.

To do so, a modification of the Maxwell-Garnett $(M M G)$ model is set forth in Sec. III, after having presented in Sec. II the deposition technique of the investigated Si nanostructures and their TEM characterization. In Sec. IV, the methodology employed in order to model the ellipsometric data is exposed. The imaginary parts of the dielectric function determined by the three different models $(M G, B E M A$, and $M M G)$ for selected Si-NPs within a silicon nitride matrix are displayed in Sec. V and discussed in Sec. VI.

\section{SYNTHESIS OF THE NANOSTRUCTURED FILMS. TEM CHARACTERIZATION AND ELLIPSOMETRIC MEASUREMENTS}

The Si-NPs grown in silicon nitride were synthesized by Plasma Enhanced Chemical Vapor Deposition (PECVD). The $\left\{\mathrm{Si}-\mathrm{NPs}+\mathrm{Si}_{3} \mathrm{~N}_{4}\right\}$ composite layers were deposited on crystalline $\mathrm{Si}$ (c-Si) substrates at a temperature of $300^{\circ} \mathrm{C}$, for several values of silane and ammonia ratio $R_{Q}=Q_{\mathrm{NH}_{3}} / Q_{\mathrm{SiH}_{4}}$. In this work, the parameter $R_{Q}$ varied between 1.00 and 1.64 . The $\mathrm{SiH}_{4}$ flow was set to a constant value of 14 SCCM (Standard Cubic Centimeter per Minute), whereas the $\mathrm{NH}_{3}$ flow was successively changed to $14,19,21,23$, and 25 SCCM. The thickness of the silicon-rich silicon nitride layers varied between 52 and $113 \mathrm{~nm}$. The $S i-N P s$ were produced after annealing at temperatures above $1000^{\circ} \mathrm{C}$, in an $\mathrm{Ar}$ or $\mathrm{N}_{2}$ atmosphere. Rapid thermal annealing $(R T A)$ and a classical tube furnace were employed in order to perform the high temperature annealing. In the following, the investigated samples are named as a function of their volume fraction of Si-NPs: $\mathrm{S}_{10.2 \%}, \mathrm{~S}_{13.7 \%}, \mathrm{~S}_{16.8 \%}, \mathrm{~S}_{19.4 \%}, \mathrm{~S}_{21.5 \%}, \mathrm{~S}_{26.6 \%}$, and $\mathrm{S}_{35.2 \%}$. All deposition parameters are summarized in Table I.

TEM images are provided for samples $\mathrm{S}_{19.4 \%}$ and $\mathrm{S}_{26.6 \%}$ in Figure 1. These micrographs unveil the presence of $\mathrm{Si}$ $N P s$. The size distributions of the Si-NPs in each sample are also plotted in the corresponding histograms. It has to be reminded that due to finite film thickness TEM images represent two dimensional projections of the characterized layer. ${ }^{6}$ Nonetheless from the histogram of Figure 1(a), it could be estimated that the mean size of the nanoparticles increases from sample $\mathrm{S}_{19.4 \%}(2 \bar{R} \approx 2.8 \mathrm{~nm})$ to sample $\mathrm{S}_{26.6 \%}$ $(2 \bar{R} \approx 4.7 \mathrm{~nm})$. Presuming a lognormal size distribution, the size dispersions $\sigma$ of the Si-NPs in samples $\mathrm{S}_{19.4 \%}$ and $\mathrm{S}_{26.6 \%}$ are evaluated to 1.25 and 1.19 , respectively. The parameter $\sigma$ is slightly smaller in sample $S_{26.6 \%}$ which can be attributed to the diverse annealing conditions (see Table I). It has to be noted that due to the resolution of the TEM, nanoparticles below a size of $2 \mathrm{~nm}$ could not be imaged. This does not exclude their possible presence in the composite layer. The arrangement of the Si-NPS within the layer shows some aggregation in both samples, although in sample $S_{19.4 \%}$, the disposition of the Si-NPs presents slightly more separated nanoparticles. The two TEM pictures reveal that the interparticle distance is relatively small (less than $5 \mathrm{~nm}$ ) and it sensitively decreases as the size (and volume fraction) of the nanoparticles increases.

A phase-modulated ellipsometer from HORIBA Scientific has been employed in order to characterize the aforementioned samples. Ellipsometry measures the variation of light polarization after reflection upon a surface. ${ }^{25}$ This polarization change is expressed by the equation:

TABLE I. Summary of the growth parameters: $R_{Q}$ is the gas flow ratio; $T_{r}$ is the annealing temperature; $A_{A}$ is the annealing ambient, and $t_{a}$ is the annealing duration. A Carbolite ${ }^{\circledR}$ tube furnace $(T F)$ was employed in order to anneal the samples $\mathrm{S}_{13.7 \%}$ and $\mathrm{S}_{19.4 \%}$ under $\mathrm{N}_{2}$ for $30 \mathrm{~min}$; whereas an $R T A$ furnace was used for the annealing of samples $\mathrm{S}_{10.2 \%}, \mathrm{~S}_{16.8 \%}, \mathrm{~S}_{21.5 \%}, \mathrm{~S}_{26.6 \%}$, $\mathrm{S}_{35.2 \%}$ under Ar for $1 \mathrm{~min}$.

\begin{tabular}{lccccc}
\hline \hline Sample & $R_{Q}$ & $T_{r}\left({ }^{\circ} \mathrm{C}\right)$ & $A_{A}$ & $t_{a}(\mathrm{~min})$ & Annealing type \\
\hline $\mathrm{S}_{10.2 \%}$ & 1.36 & 1000 & $\mathrm{Ar}$ & 1 & $R T A$ \\
$\mathrm{~S}_{13.7 \%}$ & 1.64 & 1100 & $\mathrm{~N}_{2}$ & 30 & $T F$ \\
$\mathrm{~S}_{16.8 \%}$ & 1.29 & 1000 & $\mathrm{Ar}$ & 1 & $R T A$ \\
$\mathrm{~S}_{19.4 \%}$ & 1.50 & 1100 & $\mathrm{~N}_{2}$ & 30 & $T F$ \\
$\mathrm{~S}_{21.5 \%}$ & 1.29 & 1050 & $\mathrm{Ar}$ & 1 & $R T A$ \\
$\mathrm{~S}_{26.6 \%}$ & 1.14 & 1000 & $\mathrm{Ar}$ & 1 & $R T A$ \\
$\mathrm{~S}_{35.2 \%}$ & 1.00 & 1000 & $\mathrm{Ar}$ & 1 & $R T A$ \\
\hline \hline
\end{tabular}


(a)

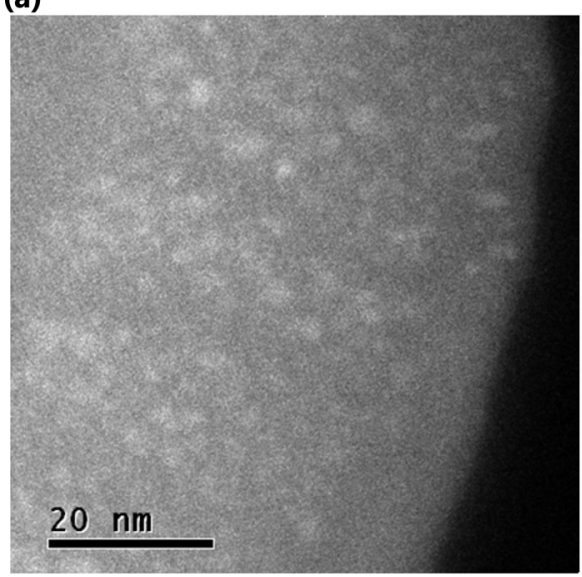

(c)

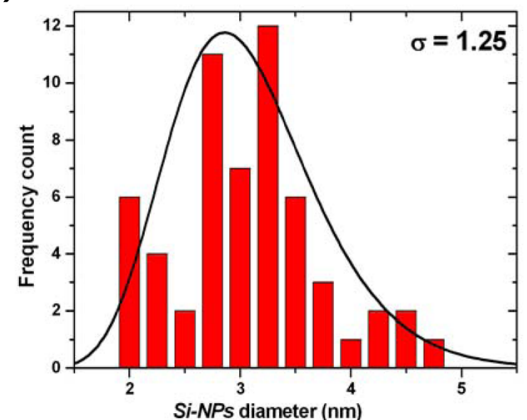

(b)

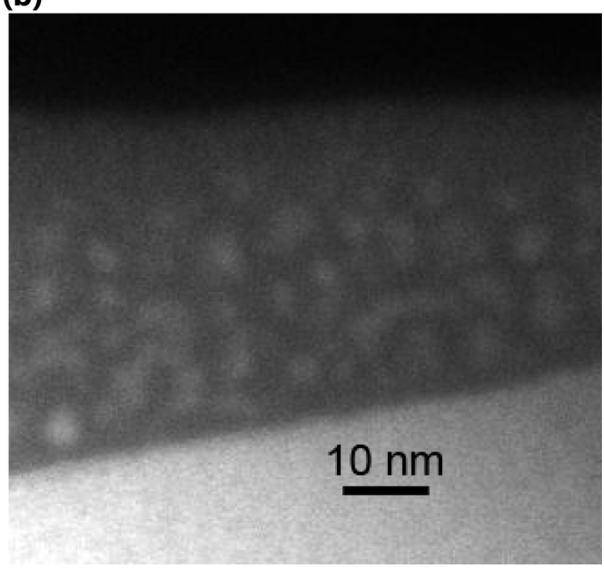

(d)

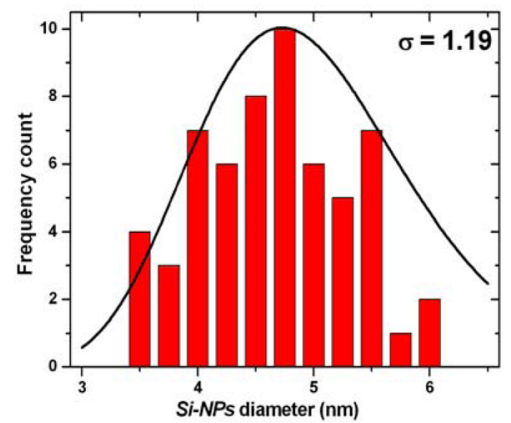

FIG. 1. Energy-filtered TEM images of samples $\mathrm{S}_{19.4 \%}$ (a) and $\mathrm{S}_{26.6 \%}$ (b). The size (diameter) distributions of the $\mathrm{Si}$ $N P s$ in each sample are displayed in the respective TEM histograms [(c) and (d)]. The Si-NPs appear as white clusters, the silicon nitride matrix is represented by a grey surface. $\frac{\tilde{r}_{p}}{\tilde{r}_{s}}=\tan \Psi \cdot e^{i \Delta}$, where $\left(\tilde{r}_{p}, \tilde{r}_{s}\right)$ are the Fresnel reflection coefficients of light, parallel and perpendicular to plane of incidence, respectively. The angle $\tan \Psi$ is the amplitude ratio, whereas the angle $\Delta$ refers to the difference in the phase between $p$ - and $s$ - polarizations of light. The configuration of this ellipsometer evaluates the parameters $\left(I_{s}, I_{c}\right)$ which are linked to $(\Psi, \Delta)$ through these relationships: $I_{s}=\sin 2 \Psi \cdot \sin \Delta$ and $I_{c}=\sin 2 \Psi \cdot \cos \Delta$. The measurement of the ellipsometric data was performed at an angle of incidence of $70^{\circ}$, at room temperature in a range of photon energy spanning from $0.60 \mathrm{eV}$ up to $6.0 \mathrm{eV}$.

\section{MODIFICATION OF MAXWELL-GARNETT EQUATION}

The optical properties of composite films such as Si-NPs embedded in silicon nitride layers are usually derived from models like Maxwell-Garnett or Bruggeman theory effective medium approximations. On the one hand, BEMA is often employed for the description of the optical properties of the silicon nitride host with inclusions of Si-NPs. ${ }^{16,25,26}$ The model is based on the following equation:

$$
f_{\mathrm{SiN}} \cdot \frac{\tilde{\varepsilon}_{\mathrm{SiN}}-\tilde{\varepsilon}_{\mathrm{eff}}}{\tilde{\varepsilon}_{\mathrm{SiN}}+2 \cdot \tilde{\varepsilon}_{\mathrm{eff}}}+f_{\mathrm{Si}-\mathrm{NPs}} \cdot \frac{\tilde{\varepsilon}_{S i-N P s}-\tilde{\varepsilon}_{\mathrm{eff}}}{\tilde{\varepsilon}_{S i-N P s}+2 \cdot \tilde{\varepsilon}_{\mathrm{eff}}}=0,
$$

where $f_{S i N}$ and $f_{S i-N P S}$ are, respectively, the volume fraction of silicon nitride and Si-NPs; $\tilde{\varepsilon}_{\mathrm{SiN}}, \tilde{\varepsilon}_{\mathrm{Si}-\mathrm{NPs}}$ and $\tilde{\varepsilon}_{\text {eff }}$ are the dielectric functions of silicon nitride, Si-NPs and the effective medium, respectively. Equation (3) is symmetric and the role of the matrix and the nanoparticles can be inverted.

On the other hand, $M G$ is usually valid in the case of well separated spherical inclusions having a low volume fraction. In such model, the interactions between the nanoparticles are described in the framework of the dipolar approximation id est higher multipole moments are neglected $^{16,25,26}$

$$
\frac{\tilde{\varepsilon}_{\text {eff }}-\tilde{\varepsilon}_{\mathrm{SiN}}}{\tilde{\varepsilon}_{\mathrm{eff}}+2 \cdot \tilde{\varepsilon}_{\mathrm{SiN}}}=f_{\mathrm{Si}-\mathrm{NPs}} \cdot \frac{\tilde{\varepsilon}_{S i-N P s}-\tilde{\varepsilon}_{\mathrm{SiN}}}{\tilde{\varepsilon}_{S i-N P s}+2 \cdot \tilde{\varepsilon}_{\mathrm{SiN}}} .
$$

This effective medium theory has been usually applied for the derivation of the dielectric function of Si-NPS. ${ }^{27}$ In the limit of small volume fractions, BEMA and $M G$ should give close results regarding the optical properties of the Si-NPS, as suggested by numerical simulations carried out by Spanoudaki and Pelster ${ }^{19}$ and $a b$ initio calculations performed by the group of Bechstedt. ${ }^{28}$

A modification of the previous $M G$ formula for a system containing nanoclusters with size distribution $P(R, \sigma)$ has been introduced. ${ }^{17,29}$ We have used such modified $M G$ $(M M G)$ formula and extended it to the case of Si-NPs embedded within a silicon nitride matrix. The corresponding $M M G$ expression is established by the following equation: ${ }^{23}$

$$
\begin{aligned}
\frac{\tilde{\varepsilon}_{\mathrm{eff}}-\tilde{\varepsilon}_{\mathrm{SiN}}}{\tilde{\tilde{\varepsilon}}_{\mathrm{eff}}+2 \cdot \tilde{\varepsilon}_{\mathrm{SiN}}}= & f_{S i-N P S} \cdot \int_{R_{\min }}^{R_{\max }} d R \cdot(R / \bar{R})^{3} \cdot P(R, \sigma) \\
& \cdot \frac{\tilde{\varepsilon}_{S i-N P S}-\tilde{\varepsilon}_{\mathrm{SiN}}}{\tilde{\varepsilon}_{S i-N P S}+2 \cdot \tilde{\varepsilon}_{\mathrm{SiN}}},
\end{aligned}
$$

where $R(\bar{R})$ is the nanocrystal (average) radius.

The parameter $f_{\text {Si-NPs }}$ used in Eqs. (3)-(5) is related to the volume fraction of Si nanoparticles that are homogeneously distributed within the layer. In case of composite layers having a rather heterogeneous topology, then the 
parameter $f_{\mathrm{Si}-\mathrm{NPs}}$ may represent only an average estimation of the volume fraction.

TEM observation of our samples has shown that $P(R, \sigma)$ can be assigned to a lognormal distribution, which has also often been observed for $\mathrm{Si}-\mathrm{NPS}$ in $\mathrm{SiO}_{2}$ layers. ${ }^{30,31}$ Indeed, the lognormal distribution describes correctly the crystallization process of solids and results from events that imply the multiplication of random variables, such as the nucleation and growth of grains with time. ${ }^{32}$ We note that $\sigma$ (unitless and greater than 1) is the standard deviation of the size distribution, also referred to as size dispersion later. The minimum $R_{\min }$ and maximum $R_{\max }$ values of the radii may be provided by $T E M$ histograms. In the numerical simulations carried out and exposed further in this work, the radii $R_{\min }$ and $R_{\max }$ were taken equal to 0.5 and $3 \mathrm{~nm}$, respectively.

Actually, the rigorous derivation of Eq. (5) should take into account the expression of a size dependence of the dielectric function $\tilde{\varepsilon}_{S i-N P s}(E, R)$ of the Si-NPs. ${ }^{17}$ However, since such an expression is not available over the whole range of photon energy explored, we are to presume that the effect of size is observable in the evolutions of the specific quantities (amplitude, broadening, transition energy, gap energy, and low frequency dielectric constant) that describe the lineshape of $\tilde{\varepsilon}_{S i-N P S}(E)$. It has to be noted that the $M M G$ model introduced in Eq. (5) obeys to the Bergman theorem [cf. Eq. (2)] since it differs from the $M G$ model by an additional coefficient that takes into account the size distribution of the $\mathrm{Si}-N P S$.

\section{DERIVATION OF THE DIELECTRIC FUNCTION OF THE SI NANOPARTICLES AND EVALUATION OF THEIR SIZE DISPERSION}

The underlying idea of Eq. (5) and the methodology presented for the evaluation of the size dispersion $\sigma$ from spectroscopic ellipsometry data, is that there should be definite values of $\sigma$ such as the dielectric functions of semiconductor nanoparticles $\tilde{\varepsilon}_{\mathrm{Si}-\mathrm{NPs}}$ derived from BEMA [Eq. (3)] and $M G$ [Eq. (4)] lead to similar line shapes. This assumption is likely to occur within a restricted photon energy range close to the absorption onset of the nanoparticles. Moreover, it extends the optical behaviors of $B E M A$ and $M G$ at the limit of small volume fractions as mentioned formerly ${ }^{19,28}$ by considering the variations induced by the size distribution of the nanoparticles.

Four different sketches are used in order to illustrate the size-distribution dependent extension of the MaxwellGarnett model (see Fig. 2). Figure 2(a) depicts a composite medium described by the classical Maxwell-Garnett approximation. The interparticle distance is large enough so that only dipolar interactions between the nanoparticles take place. Here, it is assumed that all nanoparticles have strictly the same size and are randomly dispersed in the medium.

Figures 2(b) and 2(d) illustrate two composite media with their respective size dispersions $\sigma_{1}$ and $\sigma_{2}$ such as $\sigma_{2}>\sigma_{1}$. Although the spacing between the spherical nanoparticles in these cases is substantially smaller than in schema (a); it is however assumed that the inclusions are sufficiently separated so that no aggregation of the nanoparticles occurs within the medium. For these two composite systems, the optical properties of the ensemble of nanoparticles are derived from the extension of Maxwell-Garnett (MMG) formula given by Eq. (5).

Figure 2(c) depicts a typical composite medium with an aggregated (broadly size-distributed) microstructure as usually modeled by the Bruggeman approximation. This model is adequate usually at high concentrations of nanoparticles for which case the dipole-dipole coupling approximation is no more valid. In this representation as well as in sketch (a), the size dispersion parameter $\sigma$ is unknown.

Apart from $B E M A$ and $M G$ models, Wiener showed that $\tilde{\varepsilon}_{\text {eff }}$ for a composite media of two constituents (the Si-NPS and silicon nitride matrix, in our case) varies between specific bounds that are delimited by the following interval: ${ }^{18}$

$$
\begin{aligned}
& {\left[f_{\mathrm{Si}-\mathrm{NPs}} \cdot \frac{1}{\tilde{\varepsilon}_{\mathrm{Si}-\mathrm{NPs}}}+f_{\text {mat }} \cdot \frac{1}{\tilde{\varepsilon}_{\text {mat }}}\right]^{-1} \leq \tilde{\varepsilon}_{\mathrm{eff}}} \\
& \leq f_{\mathrm{Si}-\mathrm{NPs}} \cdot \tilde{\varepsilon}_{\mathrm{Si}-\mathrm{NPs}}+f_{\text {mat }} \cdot \tilde{\varepsilon}_{\text {mat }} .
\end{aligned}
$$

Let us suppose that the quantity $\tilde{\varepsilon}_{\text {mat }}$ describing the matrix is identical (or that its variations can be neglected) in the four configurations depicted in Fig. 2. We also hypothesize that the four configurations presented in Fig. 2 have the same effective dielectric function $\tilde{\varepsilon}_{e f f}$. If this latter assumption is satisfied, then Eq. (6) shows that the ratio $f_{\mathrm{Si}-\mathrm{NPs}} / \tilde{\varepsilon}_{\mathrm{Si}-\mathrm{NPs}}$ and the product $f_{\mathrm{Si}-\mathrm{NPs}} \cdot \tilde{\varepsilon}_{\mathrm{Si}-\mathrm{NPs}}$ should vary in such a way that $\tilde{\varepsilon}_{e f f}$ remains identical in each configuration considered. In this regard, Fig. 2 suggests that the Bruggeman topology [sketch (c)] can be circumscribed by the size-distributed topologies depicted in sketches (b) and (d). By considering the parameters $\sigma_{1}$ and $\sigma_{2}$, the various sizes of the ensemble of aggregated nanoparticles can thus be recovered. Hence the dielectric function of the nanoparticles derived from $B E M A$ can be surrounded by two dielectric functions derived from (a)

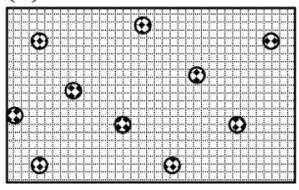

(b)

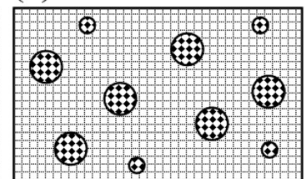

(c)

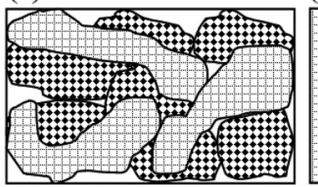

(d)

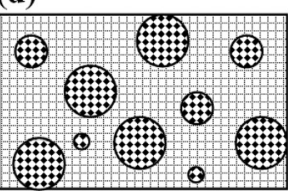

FIG. 2. The various sketches used for the interpretation of the size-distribution dependent extension of the Maxwell-Garnett model presented in this work. (a) A composite medium of isolated nanoparticles described by the classical Maxwell-Garnett theory; (b) A composite medium with a given size dispersion $\sigma_{1}$; (c) A composite medium represented by the Bruggeman approximation which refers to an aggregated microstructure of the nanoparticles; and (d) A composite medium with a larger size dispersion $\sigma_{2}>\sigma_{1}$. In cases (b) and (d), the interparticle distances are nonzero but significantly smaller than in case (a). The matrix is represented by the grid and the volume of the $S i$-NPs is delimited by diamonds. It is assumed that all four configurations have the same effective dielectric function $\tilde{\varepsilon}_{e f f}$ that is represented here by the area of the rectangle delimiting the composite system $\{$ matrix $+S i-N P S\}$. Note that the relative dimensions of the Si-NPs have been emphasized for better visualization. 
(a)
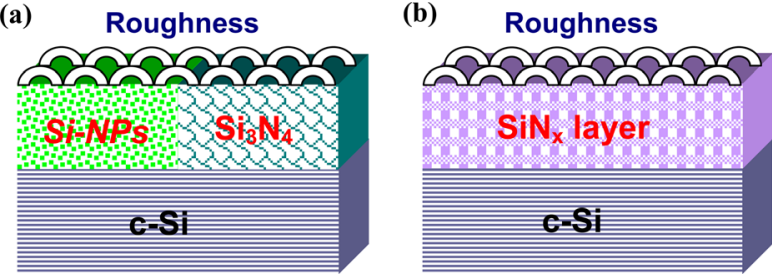

FIG. 3. The two optical models employed for the derivation of the dielectric function of the $S i-N P s$ and evaluation of their size dispersion. The optical model (a) describes the same layer as a composite $\left\{S i-N P S+\mathrm{Si}_{3} \mathrm{~N}_{4}\right\}$ film by taking into account the optical contributions of the Si-NPs and the silicon nitride matrix distinctly. The optical model (b) considers the silicon-rich silicon nitride $\mathrm{SiN}_{\mathrm{x}}$ film as a homogeneous layer. The same optical models as those we used in Ref. 34 are employed.

$M M G$ with distinct size dispersion parameters. We will show in Sec. V that this may indeed be possible within a spectral range $I_{E}$ close to the absorption onset of the Si-NPs.

In the optical model of Fig. 3(a), two layers are used above the substrate: the first one corresponds to the siliconrich silicon nitride composite layer which contains the $\mathrm{Si}$ $N P S$ embedded in the silicon nitride matrix; and the second one, the surface roughness of the specimen. The optical properties of the roughness are modeled with a mixture consisting in 50 vol. \% the underneath layer and 50 vol. \% void and modeled by BEMA. In this optical model, the parameters adjusted are the thicknesses of the composite film and its corresponding roughness along with the volume fraction of the nanoparticles. Then the ellipsometric data are fitted in a reduced energy range $[0.6 \mathrm{eV}-2.0 \mathrm{eV}]$. To do so a mixture between the optical properties of amorphous ( $a$-Si) and crystalline $\mathrm{Si}$ is considered as shown in Table II. It points out that the investigated nanoparticles are mainly amorphous. Particularly from sample $S_{10.2 \%}\left(f_{c-S i}=4.64 \%\right)$ to sample $\mathrm{S}_{26.6 \%}\left(f_{c-S i}=4.76 \%\right)$, the consideration of crystalline $\mathrm{Si}$ is necessary in order to obtain the small values of $\chi^{2}$ as displayed. This indicates the presence of a non-negligible amount of crystallized nanoparticles in the composite layer. The advantage of the optical model of Fig. 3(a) is that it enables a worthwhile estimation of the respective proportions of crystalline and amorphous nanoparticles, which seems difficult by using other techniques. Note that the optical constants of the silicon nitride matrix $\left(\tilde{\varepsilon}_{\mathrm{SiN}}\right)$ can be either measured separately on a sample without $S i-N P S$ or taken from the referenced data in the literature as done in this

TABLE II. Values of film thickness $t$ and proportion of amorphous $\left(f_{a-S i}\right)$ and crystalline $\left(f_{c-S i}\right)$ Si nanoparticles evaluated for each investigated sample in the reduced energy range [0.6-2.0 eV]. The respective total volume fraction of $S i-N P S\left(f_{S i-N P S}\right)$ is also provided. The corresponding $\chi_{\text {red }}^{2}$ over this reduced energy range are also given.

\begin{tabular}{lccccc}
\hline \hline Sample & $t(\mathrm{~nm})$ & $f_{a-S i}(\%)$ & $f_{c-S i}(\%)$ & $f_{S i-N P S}(\%)$ & $\chi_{\text {red }}^{2}$ \\
\hline $\mathrm{S}_{10.2 \%}$ & 53.6 & 5.56 & 4.64 & 10.2 & 0.37 \\
$\mathrm{~S}_{13.7 \%}$ & 113.2 & 6.88 & 6.80 & 13.7 & 1.25 \\
$\mathrm{~S}_{16.8 \%}$ & 52.9 & 11.8 & 4.97 & 16.8 & 0.75 \\
$\mathrm{~S}_{19.4 \%}$ & 108.8 & 14.7 & 4.72 & 19.4 & 1.30 \\
$\mathrm{~S}_{21.5 \%}$ & 52.8 & 18.4 & 3.14 & 21.5 & 0.62 \\
$\mathrm{~S}_{26.6 \%}$ & 57.4 & 21.8 & 4.76 & 26.6 & 0.38 \\
$\mathrm{~S}_{35.2 \%}$ & 43.6 & 22.5 & 12.7 & 35.2 & 0.06 \\
\hline \hline
\end{tabular}

work. In the following simulations, the value of $f_{S i-N P s}$, for each sample, is the same in Eqs. (3)-(5).

The effective dielectric function $\tilde{\varepsilon}_{\text {eff }}$ of the system $\{$ matrix + Si nanoparticles $\}$ is calculated by using the optical model depicted in Fig. 3(b). Such layer structure considers the whole composite medium as a homogeneous layer. In that second optical model, only the appropriate thicknesses are adjusted before deriving the optical constants $\tilde{\varepsilon}_{\text {eff }}$ of the $\mathrm{SiN}_{\mathrm{X}}$ layer. The function $\tilde{\varepsilon}_{\text {eff }}$ is derived by using either a dispersion formula (with multiple oscillators) or without the use of any fitting parameter by wavelength-by-wavelength $(\lambda$-by- $\lambda)$ numerical inversion. We have demonstrated before that the line shape of the effective dielectric function $\tilde{\varepsilon}_{e f f}$ of silicon-rich silicon oxide/nitride films can be characterized by some features located at specific photon energies close to the critical points of crystalline silicon (between $3.3 \mathrm{eV}$ and $5.5 \mathrm{eV}$ ). Therefore a cautious examination of $\tilde{\varepsilon}_{e f f}$ should be helpful in predicting the transition energies associated to the dielectric function of the nanoparticles. ${ }^{23,24} \mathrm{Next}$, the spectra of $\tilde{\varepsilon}_{e f f}$ corresponding to the seven investigated specimens are not displayed and attention will rather be given to the dielectric function $\tilde{\varepsilon}_{\mathrm{Si}-\mathrm{NPs}}$ of the nanoparticles. We will compare the line shapes obtained from $M G, B E M A$ and $M M G$.

The dielectric function of the nanoparticles $\tilde{\varepsilon}_{\mathrm{Si}-\mathrm{NPs}}$ represents here the unknown quantity and can be numerically evaluated using Eqs. (3)-(5). The values of $\tilde{\varepsilon}_{\mathrm{Si}-\mathrm{NPs}}$ may be calculated using dispersion formulas such as ForouhiBloomer, ${ }^{27}$ Tauc-Lorentz ${ }^{10,33}$ or alternatively by $\lambda$-by- $\lambda$ numerical inversion. ${ }^{30,31}$ We have previously evidenced similarities and differences existing between these models and methods of derivation. ${ }^{34}$ In particular, the results presented in this paper are derived from numerical inversion of the ellipsometric data. The dielectric functions related to the ensemble of Si-NPs, displayed afterwards in Fig. 6, are deduced from the same effective dielectric function. Based on the very small $\chi^{2}$ obtained we make the assumption that the assessed $\tilde{\varepsilon}_{e f f}$ corresponding to a given sample is the most accurate effective dielectric function. The $\tilde{\varepsilon}_{\text {Si-NPs }}$ computed for the composite media of Figs. 2(b) and 2(d) correspond to

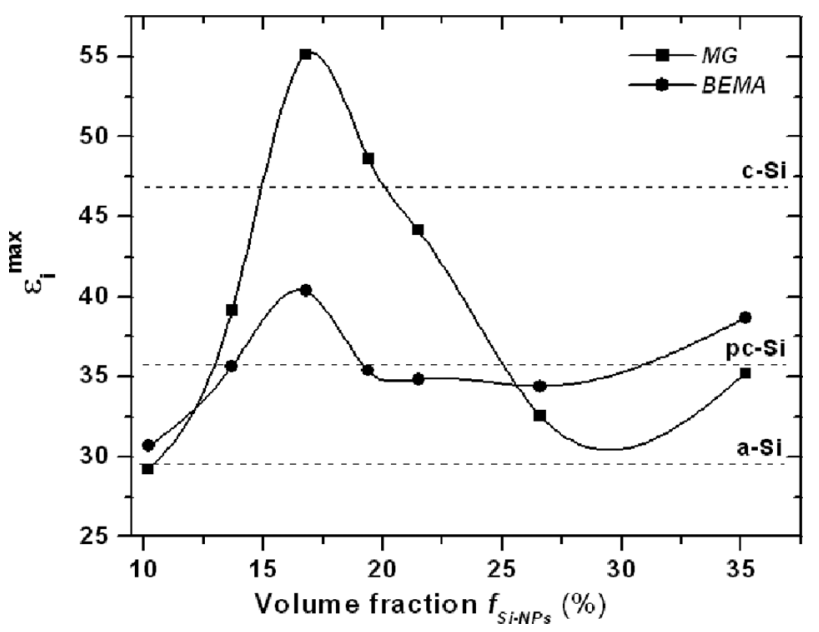

FIG. 4. Evolution of the amplitude $\varepsilon_{i}^{\max }$ of the $\varepsilon_{i}$ peak derived from $M G$ and $B E M A$ for the samples studied in this work. The values of $\varepsilon_{i}^{\max }$ related to the main peak of $a-S i, p c-S i$, and $c-S i$ are also provided as references (dashed lines). The spline lines connecting each data are guides for the eye. 
the dielectric functions of an ensemble of Si-NPs with volume fraction $f_{\mathrm{Si}-\mathrm{NPs}}$ and size dispersion equal to $\sigma_{1}$ and $\sigma_{2}$, respectively.

We derived the optical constants of the nanoparticles by numerical inversion. Thus there are only two parameters of the model which may correlate with the size dispersion extracted: the film thickness and the size of the nanoparticles via their volume fraction. On the one hand, since the thicknesses of the samples are larger than the average sizes of the nanoparticles, we can minimize the correlations between the film thicknesses with the variations of the size dispersion parameter. This is the case provided that the thicknesses are accurately determined before calculating the optical constants [as displayed in Table II by using the optical model of Fig. 3(a)].

On the other hand, more significant correlations are expected between the volume fraction $\left(f_{\mathrm{Si}-\mathrm{NPs}}\right)$ of the nanoparticles (indirectly connected to their average size) and their size dispersion. For a sample with a specific average size of the Si nanoparticles, one can have an idea about the correlation between the volume fraction and the size dispersion by analyzing the size distribution histogram obtained from TEM (see Fig. 1). Indeed the volume fraction of the nanoparticles will appreciably differ whether it is assumed that all nanoparticles have strictly the same size $(\sigma \rightarrow 1)$ or have a precise size distribution $(\sigma>1)$ peaking at their average size. In this latter case, the larger the size dispersion, the stronger will be the correlation with the volume fraction of the nanoparticles. However, it is not our goal here to derive values for the corresponding correlation coefficients. In the methodology presented in this work, the parameters $\left(f_{\mathrm{Si}-\mathrm{NPs}}\right)$ and $\sigma$ are evaluated separately in order to minimize the crosscorrelation.

\section{RESULTS}

Now we shall present the imaginary dielectric functions derived from the various models ( $M G, B E M A$ and $M M G)$ for the investigated samples. In Refs. 23 and 24, it has been shown that the real part of the dielectric function is also subjected to similar evolutions with the change of the parameter $\sigma$. However we focus on $\varepsilon_{i}$ here.

The evolution of the peak $\varepsilon_{i}^{\max }$ of the imaginary dielectric function calculated from the $M G$ and BEMA models for the seven samples studied here are presented in Figure 4. The amplitudes of $\varepsilon_{i}$ derived from BEMA generally evolve between those of $a-S i \quad\left(\varepsilon_{i}^{\max }=29.2\right)$ and polycrystalline $S i \quad\left(\varepsilon_{i}^{\max }\right.$ $=35.6$ ). In sample $S_{10.2 \%}$ with the smallest concentration of Si-NPS $\left(f_{S i-N P s}=10.2 \%\right)$, the value of $\varepsilon_{i}^{\max }\left(\varepsilon_{i}^{\max }=46.7\right)$ is close to that of $a-S i$. The quantity $\varepsilon_{i}^{\text {max }}$ increases and gets closer to or higher than that of polycrystalline $S i$, due to higher concentrations of crystallized Si-NPs from sample $\mathrm{S}_{13.7 \%}$ $\left(\varepsilon_{i}^{\max }=35.7\right)$ to sample $\mathrm{S}_{35.2 \%}\left(\varepsilon_{i}^{\max }=38.7\right)$.

Figure 4 also shows the evolution of the dielectric function computed from Maxwell-Garnett approximation. A clear increase of the magnitude of $\varepsilon_{\mathrm{i}}$ is noticed from sample $\mathrm{S}_{10.2 \%}$ to sample $\mathrm{S}_{16.8 \%}$ : in the latter specimen the maximum value of $\varepsilon_{\mathrm{i}}$ is 52 at $3 \mathrm{eV}$, whereas it reaches only 26 for sample $S_{10.2 \%}$. Thereafter, this value gradually decreases with the volume fraction. The maximum value of the dielectric function, calculated using $M G$ model exceeds that of bulk crystalline in $\mathrm{Si}$ for samples $\mathrm{S}_{16.8 \%}$ and $\mathrm{S}_{19.4 \%}$. On the contrary, the values determined from BEMA retain some physical meaning. Knowing that in a given composite layer, the same initial effective dielectric function has been used for both BEMA and $M G$ models (cf. Sec. IV), each model provides a precise lineshape for $\varepsilon_{i}$ that accommodates to the characteristic topology of the Si-NPs $\left(f_{S i-N P S}\right)$ and to their environment $\left(\tilde{\varepsilon}_{\mathrm{SiN}}\right)$. The extremely high values of the amplitude of $\varepsilon_{i}$ are indications of the application limit of the $M G$ model-owed to its characteristic dipolar approximation-to the $S i-N P s$ in the investigated samples. In samples $\mathrm{S}_{16.8 \%}$, $S_{19.4 \% \text {, and }} S_{21.5 \%}$, the phase separation between the silicon nitride and the excess silicon may have led to Si-NPs with irregular (elongated) shapes, as already observed for other Si-NPs synthesized in a similar way. ${ }^{35}$

Another important quantity related to the lineshape of $\varepsilon_{i}$ is given by the position energy of the maximum of absorption. In case of $M G$ model, a continuous redshift of the peak energy $E^{\max }$ of $\varepsilon_{\mathrm{i}}$ is observed as $f_{\text {Si-NPS }}$ increases from sample $\mathrm{S}_{10.2 \%}\left(E^{\max } \approx 3.50 \mathrm{eV}\right)$ to sample $\mathrm{S}_{21.5 \%}\left(E^{\max } \approx 2.95 \mathrm{eV}\right)$ (see Fig. 5). The values of $E^{\max }$ rise for samples $\mathrm{S}_{26.6 \%}$ and $\mathrm{S}_{35.2 \%}$. However, the values of $E^{\mathrm{max}}$ calculated from $M G$ formula are significantly lower than that of amorphous $\mathrm{Si}$ $\left(E^{\max } \approx 3.75 \mathrm{eV}\right)$, which is another clue evidencing the limitation of MG for the samples studied here. This comes from the fact that the Si-NPs cannot be considered as isolated spheres: manifestly, the size of the nanoparticles is no longer much smaller than the interparticle distance and consequently aggregation occurs in our composite systems, as evidenced in Fig. 1. Regarding the BEMA model, the same figure shows that $E^{\max }$ evolves between the corresponding values for $a-S i$ and $p c-S i\left(E^{\max } \approx 3.75 \mathrm{eV}\right)$ : however, unlike the $M G$ model, there is with $B E M A$ no well defined trend that could be pointed out from the variations of $E^{\max }$ observed from sample $S_{10.2 \%}$ to $S_{21.5 \%}$.

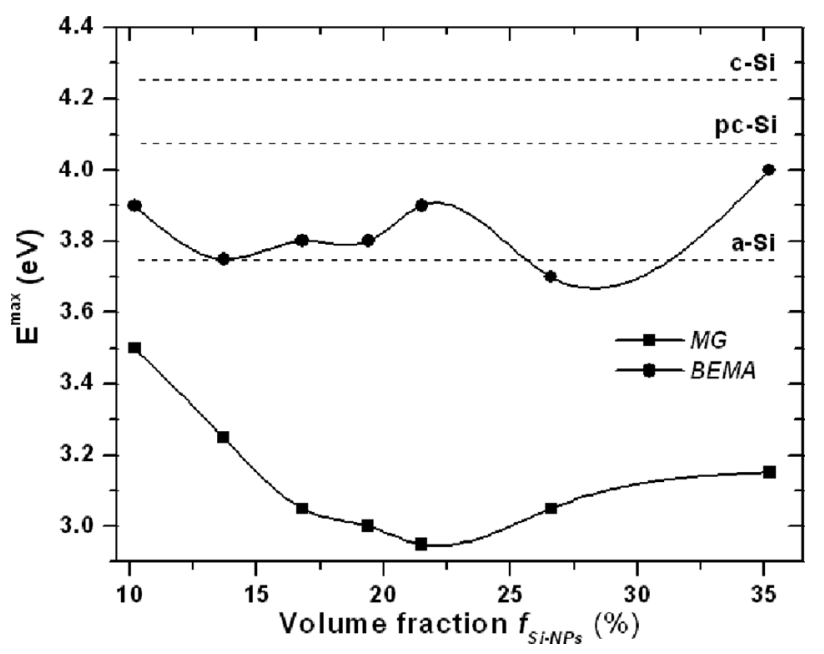

FIG. 5. Evolution of the position $E^{\max }$ of the $\varepsilon_{i}$ peak obtained from $M G$ and $B E M A$ for the samples investigated in this work. The values of $E^{\text {max }}$ related to the main peak of amorphous $\mathrm{Si}(a-\mathrm{Si})$, polycrystalline $\mathrm{Si}(p c-\mathrm{Si})$ and crystalline $\mathrm{Si}(c-\mathrm{Si})$ are also provided as references (dashed lines). The spline lines connecting each data are guides for the eye. 
The evolutions of $\varepsilon_{i}^{\max }$ and $E^{\max }$ indicate that $M G$ model fails in describing correctly the optical constants of the $\mathrm{Si}$ $N P s$. On the contrary, BEMA model appears to provide realistic lineshapes for the imaginary dielectric functions. Next, it will be shown that the $\varepsilon_{i}$ calculated with $M M G$ model support the lineshapes obtained from BEMA, and enable deducing information about the size dispersion of the Si-NPs within a restricted range close to their absorption onset. Figure 6 compares the imaginary dielectric functions of $\mathrm{Si}$ $N P s$ calculated from samples $\mathrm{S}_{10.2 \%}$ to $\mathrm{S}_{35.2 \%}$, obtained with $B E M A, M G$, and $M M G$ models. In the latter one, different values of standard deviation $\sigma$ (varying between 1.05 and 1.35 ) of the lognormal size distribution are taken into account. The imaginary dielectric functions derived from the aforementioned models show featureless line shapes. This observation is in good agreement with the dominant fraction of amorphous Si-NPs as evidenced by the results of Table II. Expectedly this figure shows that the $\varepsilon_{i}$ spectra derived from Maxwell-Garnett and Bruggeman theories are rather dissimilar. The discrepancies are small when the volume fraction of the nanoparticles is not high such as in sample $\mathrm{S}_{10.2 \%}$. The gap between $B E M A$ and $M G$ becomes more significant as $f_{S i-}$ ${ }_{N P S}$ increases up to $35.2 \%$ for sample $\mathrm{S}_{35.2 \%}$. At such elevated concentrations the microtopology of the ensemble of $\mathrm{Si}-\mathrm{NPS}$ is featured by aggregation which explains the deviations thus observed.

Moreover, when the value of $\sigma$ in the $M M G$ model gradually increases, we see a reduction in the amplitude of $\varepsilon_{i}$. Interestingly, each scheme of Fig. 6 shows the presence of two values of $\sigma\left(\sigma_{\min }\right.$ and $\left.\sigma_{\max }\right)$ for which the corresponding
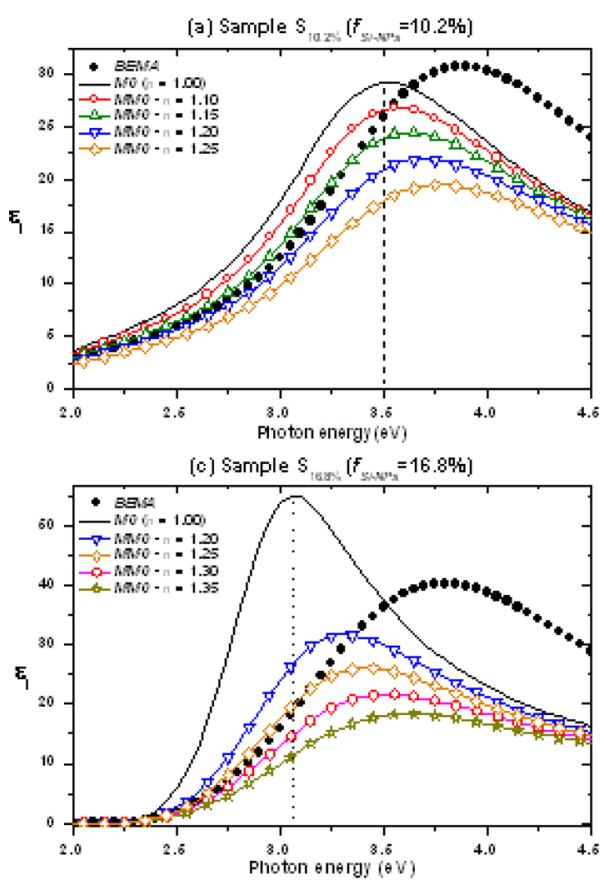

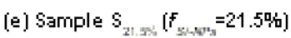

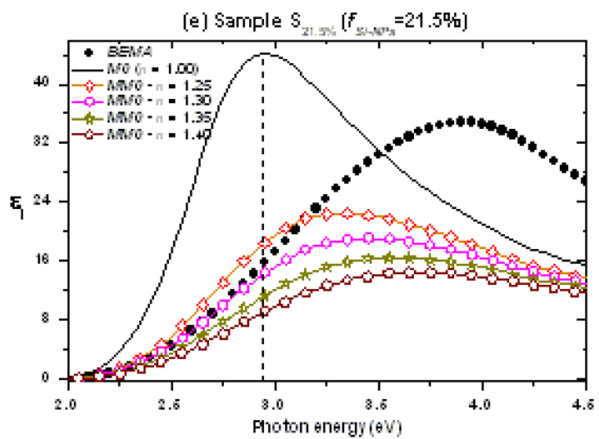

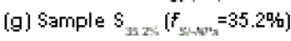

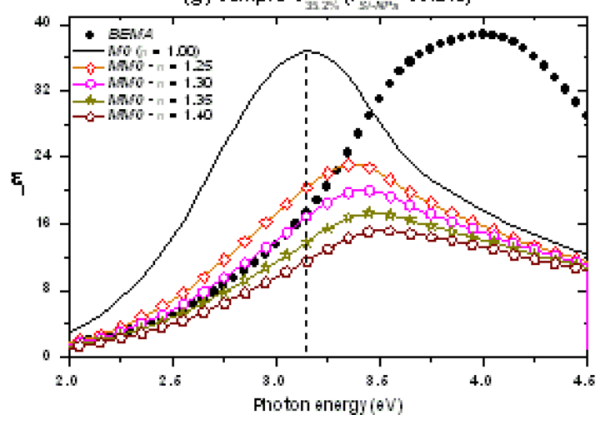

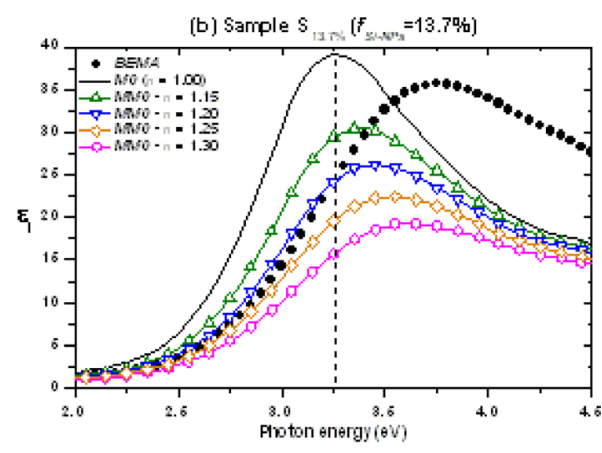
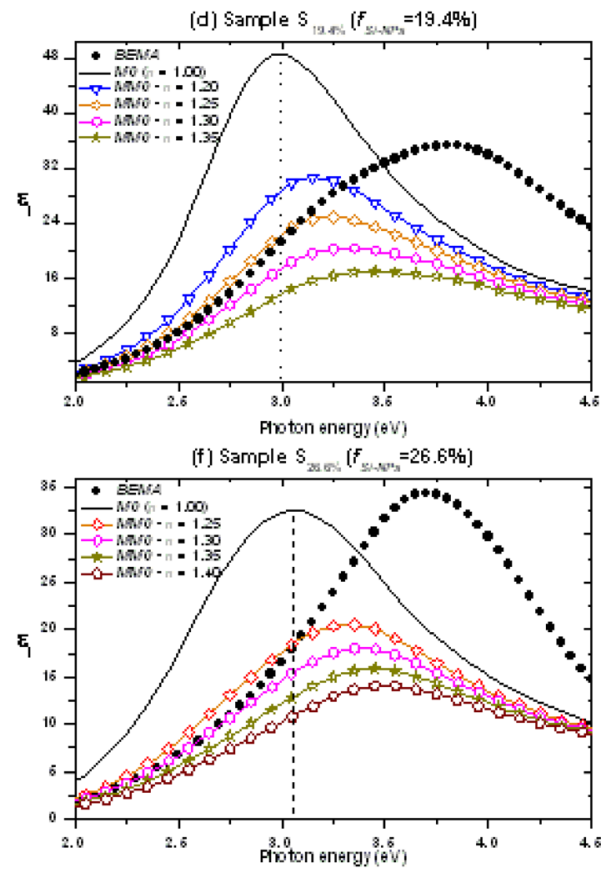

FIG. 6. Comparison between the imaginary part of the dielectric functions of Si-NPs calculated by BEMA, MG and $M M G$ for different values of $\sigma$ assuming a lognormal size distribution. The vertical dashed lines represent the resonance peak of $\varepsilon_{i}$ derived from $M G$. 
dielectric functions surround the imaginary dielectric function calculated by Bruggeman over a relatively wide photon energy range comprised between $2 \mathrm{eV}$ and $3 \mathrm{eV}$. The amplitudes of the imaginary dielectric function of the Si-NPs are generally underestimated in the spectra calculated by $M M G$ model. In addition the $\varepsilon_{i}$ derived from $M M G$ are always blueshifted relatively to $M G$ model and red-shifted comparatively to Bruggeman.

Although several curves plotted in Fig. 6 are not relevant according to the optical criterion (Eq. (6), for instance), the choice has intentionally been made to display the lineshapes of $\varepsilon_{i}$ calculated from $M M G$ with four different values of $\sigma$. By doing so we want to emphasize that the lineshape of $\varepsilon_{i}$ derived from BEMA is always surrounded by only two distinct lineshapes deduced from $M M G$. The values of $\sigma_{\min }$ and $\sigma_{\max }$ evaluated for each sample are given in Table III. Table III indicates that $\sigma_{\min }$ and $\sigma_{\max }$ continuously increase with the volume fractions of the Si-NPs. However one can notice that, from samples $S_{16.8 \%}$ to $S_{26.6 \%}$, the values obtained for $\left[\sigma_{\text {min }}-\sigma_{\text {max }}\right]$ remain in the range $[1.25-1.30]$. This result does not mean that the size dispersion is constant in these four samples; but due to the step of 0.05 that has been chosen for the variation of $\sigma$, the corresponding precise values are not presented here.

As $\sigma$ increases a broadening of the $\varepsilon_{i}$ peak calculated from $M M G$ is also observed in Fig. 6. This behavior is consistent with the influence of size dispersion which contributes to the inhomogeneous broadening of an absorption peak. ${ }^{17,23}$ On the contrary, the BEMA and $M G$ lineshapes do not reveal any visible manifestation of size distribution on the corresponding spectra of $\varepsilon_{i}$. The spectra from $0.6 \mathrm{eV}$ up to $2.0 \mathrm{eV}$ (not shown in Fig. 6) indicate that there is no perceptible difference between the lineshapes of $\varepsilon_{i}$ computed by $M G, B E M A$ and $M M G$. This is due to the transparency of the $\mathrm{Si}-N P s$ to the incident photons since the average gap energy of the nanoparticles varies between $1.3 \mathrm{eV}$ and $2.0 \mathrm{eV}$. Above this latter energy, Fig. 6 indicates the influence of size dispersion on the imaginary dielectric function, which can be expected. At photon energies higher than $3 \mathrm{eV}$, the large differences observed between $B E M A$ and $M M G$ models can be explained by the influence of multipolar interactions (owed to aggregation of the nanoparticles) which increase when the interparticle distance drastically decreases.

TABLE III. Values of the standard deviations $\sigma_{\min }$ and $\sigma_{\max }$ of $M M G$ model used to surround the imaginary dielectric functions calculated by BEMA in the photon energy range $I_{E}$. It is interesting to notice that the upper limit of $I_{E}$, which is given by the higher value of size dispersion $\sigma_{\max }$, is close to the resonance peak of $\varepsilon_{i}$ computed from $M G$ (cf. Fig. 6). The volume fractions of the $S i-N P s\left(f_{S i-N P s}\right)$ are also recalled.

\begin{tabular}{lcccc}
\hline \hline Sample & $f_{S i-N P S}(\%)$ & $\sigma_{\min }(\varnothing)$ & $\sigma_{\max }(\varnothing)$ & $I_{E}(\mathrm{eV})$ \\
\hline $\mathrm{S}_{10.2 \%}$ & 10.2 & 1.15 & 1.20 & $2.00-3.35$ \\
$\mathrm{~S}_{13.7 \%}$ & 13.7 & 1.20 & 1.25 & $2.00-3.25$ \\
$\mathrm{~S}_{16.8 \%}$ & 16.8 & 1.25 & 1.30 & $2.00-3.15$ \\
$\mathrm{~S}_{19.4 \%}$ & 19.4 & 1.25 & 1.30 & $2.00-3.10$ \\
$\mathrm{~S}_{21.5 \%}$ & 21.5 & 1.25 & 1.30 & $2.00-3.15$ \\
$\mathrm{~S}_{26.6 \%}$ & 26.6 & 1.25 & 1.30 & $2.00-3.15$ \\
$\mathrm{~S}_{35.2 \%}$ & 35.2 & 1.30 & 1.35 & $2.00-3.05$ \\
\hline \hline
\end{tabular}

The effective number of electrons $n_{\text {eff }}$ is linked to the first order of the momentum of the imaginary dielectric function. The quantity $n_{\text {eff }}$ has been derived using the following equation: ${ }^{30}$

$$
n_{\mathrm{eff}}(E)=\frac{2}{\pi} \cdot \frac{m_{e} \cdot \varepsilon_{0}}{\hbar^{2} \cdot N_{A} \cdot e^{2}} \int_{E_{i}}^{E_{f}} d E^{\prime} E^{\prime} \cdot \varepsilon_{i}\left(E^{\prime}\right),
$$

where $m_{e}$ and $e$ are, respectively, the charges and mass of the electron; $\hbar$ is the reduced Planck constant; $\varepsilon_{0}$ is the dielectric permittivity of vacuum; $N_{A}$ is the Avogadro constant; $E$ is the photon energy and $E^{\prime}$ is a blank variable with the dimension of energy. The dielectric function of the Si-NPs should not only satisfy the Kramers-Kronig relationships, but it should also obey to the sum rule given by Eq. (7). $E_{i}$ and $E_{f}$ used represent the upper and lower limits of the integral of Eq. (7). As well as $I_{E}$, the interval $\left[E_{i}-E_{f}\right]$ defines the largest energy range over which the $\varepsilon_{i}$ derived from $B E M A$ is surrounded by two distinct lineshapes calculated with $M M G$. In our calculations we have taken for $E_{i}$ and $E_{f}$ the figures given in Table III. It may also be possible to take a value lower than $2.0 \mathrm{eV}$ for $E_{\mathrm{i}}$ : this modification changes only slightly the value of $n_{\text {eff }}$, but the result regarding the surrounding performed with $M M G$ remains unaffected. On the contrary, the value of $E_{f}$ is better defined and is more specific to a given ensemble of $S i$-NPs than $E_{i}$. It has to be mentioned that consideration of the effective number of electrons was done by Gallas et al. only. ${ }^{30}$

The figures of $n_{\text {eff }}$ computed for the seven investigated specimens are collected in Table IV. The effective number of electrons is derived from the line shapes of $\tilde{\varepsilon}_{\mathrm{Si}-\mathrm{NPs}}$ given by $M G, B E M A$ and $M M G$ with different values of the size dispersion $\sigma$. In general, the $M G$ model gives higher values of $n_{\text {eff }}$ than BEMA. In the case of $M M G$, the effective number of electrons of the nanoparticles decreases as $\sigma$ increases from 1.10 to 1.40 . This is due to a shift of the line shape of $\tilde{\varepsilon}_{\mathrm{Si}-\mathrm{NPs}}$ as displayed in Fig. 4. In the $[2.00 \mathrm{eV}-3.35 \mathrm{eV}]$ energy range, crystalline $\mathrm{Si}$ has a lower value of $n_{\text {eff }}(0.114)$ than amorphous $\mathrm{Si}(0.431)$. Hence the decrease of $n_{\text {eff }}$ with $\sigma$ can be interpreted as the contribution of the large nanoparticles that are likely to be more crystallized than the small ones. Above a certain value of size dispersion $(\sigma>1.40)$, the effective number of electrons gets too small in comparison to the figures derived from $M G$ and $B E M A$. Therefore, it may probably become unphysical for the investigated Si-NPs. Table IV reveals us that there are, for all studied specimens, two values of $\sigma$ (from the $M M G$ model) which circumscribe the figure of $n_{\text {eff }}$ deduced from $B E M A$.

\section{DISCUSSION}

The results obtained for the series of samples investigated here reveal that two factors may influence the optical constants of the Si-NPs calculated by ellipsometry: (i) the size dispersion and (ii) the optical constants variations with size. In this regard, Fig. 3 and Table II of Ref. 23 suggest that both factors affect at the same time the optical response of an ensemble of $\mathrm{Si}$-NPs. On the one hand, the consideration of the effect of size dispersion implies that each specific size 
TABLE IV. Values of the effective number of electrons $n_{\text {eff }}$ derived for samples $\mathrm{S}_{10.2 \%}$ to $\mathrm{S}_{35.2 \%}$. The data are calculated in the energy range $I_{E}=\left[E_{i}, E_{f}\right]$ (cf. Table III) from $M G, B E M A$, and $M M G$ with diverse values of size dispersion $\sigma$. For each sample, the values of $n_{\text {eff }}$ in bold delimit the estimated range for the size dispersion of the Si-NPs. For comparisons, the values of $n_{\mathrm{eff}}$ for $a-S i, c-S i$ and $p c-S i$ are, respectively, equal to $0.431,0.114$, and 0.198 in the $[2.00 \mathrm{eV}-3.35 \mathrm{eV}]$ energy range.

\begin{tabular}{|c|c|c|c|c|c|c|c|c|}
\hline \multicolumn{2}{|l|}{ EMA } & \multicolumn{7}{|c|}{$n_{e f f}(\varnothing)$} \\
\hline Name & $\sigma(\varnothing)$ & Sample $\mathrm{S}_{10.2 \%}$ & Sample $\mathrm{S}_{13.7 \%}$ & Sample $S_{16.8 \%}$ & Sample $S_{19.4 \%}$ & Sample $\mathrm{S}_{21.5 \%}$ & Sample $S_{26.6 \%}$ & Sample $S_{35.2 \%}$ \\
\hline$M G$ & 1.00 & 0.436 & 0.491 & 0.601 & 0.710 & 0.667 & 0.557 & 0.434 \\
\hline$M M G$ & 1.10 & 0.386 & - & - & - & - & - & - \\
\hline$M M G$ & 1.15 & 0.340 & 0.344 & - & - & - & - & - \\
\hline$M M G$ & 1.20 & 0.292 & 0.278 & 0.265 & 0.394 & - & - & - \\
\hline$M M G$ & 1.25 & 0.248 & 0.222 & 0.196 & 0.308 & 0.267 & 0.286 & 0.209 \\
\hline$M M G$ & 1.30 & - & 0.179 & 0.146 & 0.242 & 0.208 & 0.235 & 0.171 \\
\hline$M M G$ & 1.35 & - & - & 0.112 & 0.193 & 0.165 & 0.196 & 0.141 \\
\hline$M M G$ & 1.40 & - & - & - & - & 0.134 & 0.165 & 0.118 \\
\hline BEMA & - & 0.317 & 0.251 & 0.177 & 0.282 & 0.226 & 0.253 & 0.158 \\
\hline
\end{tabular}

present in the distribution brings its own contribution to the optical constants calculated for the ensemble of Si-NPS. Therefore such an effect can be analyzed by comparing composite layers containing $\mathrm{Si}-\mathrm{NPS}$ with average sizes that are ideally identical or, in most common cases, close to each other: for instance, in this study, samples $\mathrm{S}_{19.4 \%}(\sigma \approx 1.27)$ and $S_{21.5 \%}(\sigma \approx 1.29)$. Figure 4 , as well as Figs. $6(d)$ and $6(\mathrm{e})$, suggest that the corresponding amplitudes of $\varepsilon_{i}(\approx 35.4$ and $\approx 34.8$ in samples $S_{19.4 \%}$ and $S_{21.5 \%}$, respectively; for the lineshapes derived from $B E M A$ ) are close to each other. The variation of the transition energies from $3.8 \mathrm{eV}$ (sample $\mathrm{S}_{19.4 \%}$ ) to $3.9 \mathrm{eV}$ (sample $\mathrm{S}_{21.5 \%}$ ) is not significant. It is interesting to note that the corresponding curvatures of $\varepsilon_{i}$ in the $[2.0 \mathrm{eV}-3.0 \mathrm{eV}]$ range (see Fig. 6, in the case of BEMA) slightly change when $\sigma$ increases. Besides, the effect of size dispersion appears to be also visible on the broadening of $\varepsilon_{i}$ : indeed the full width at half maximum of $\varepsilon_{i}$ derived from $B E M A$ is near to $2.15 \mathrm{eV}$ (sample $\mathrm{S}_{19.4 \%}$ ) and $2.2 \mathrm{eV}$ (sample $\mathrm{S}_{21.5 \%}$ ). This observation is coherent with what can be expected from the inhomogeneous broadening due to size dispersion. The results presented here reveal the good sensitivity of ellipsometry to the size dispersion of the Si-NPs.

On the other hand, the influence of the optical constants variations with size on the calculated optical constants can unambiguously be revealed only in the case of composite layers having nanoparticles with identical size dispersions. The ideal case would be the investigation of a series of samples in which the Si-NPs have no or very narrow size dispersion ( $\sigma \cong 1$, in a log-normal distribution). In order to illustrate that second issue, the lineshapes obtained for samples $S_{21.5 \%}$ and $S_{26.6 \%}$ are found to represent the best possible comparison in our study. We have derived that $\sigma \approx 1.29$ and 1.28 for the nanoparticles in $\mathrm{S}_{21.5 \%}$ and $\mathrm{S}_{26.6 \%}$, respectively. A redshift of the peak energy is noticed from $3.9 \mathrm{eV}$ (sample $\mathrm{S}_{21.5 \%}$ ) to $3.7 \mathrm{eV}$ (sample $\mathrm{S}_{26.6 \%}$; see Fig. 5 in the case of BEMA). Such a redshift was previously observed when increasing the size of the Si-NPs. ${ }^{30,31}$ Besides, a small change in the curvature of $\varepsilon_{i}$ in the $[2.0 \mathrm{eV}-3.0 \mathrm{eV}]$ range between these two samples can be noted. Interestingly the broadening of $\varepsilon_{i}$ derived from $B E M A$ is appreciably reduced from $\mathrm{S}_{21.5 \%}(\approx 2.2 \mathrm{eV})$ to $\mathrm{S}_{26.6 \%}(\approx 1.4 \mathrm{eV})$. We attribute this to the crystallization of a larger number of Si-NPs: indeed Table II indicates that the relative volume fraction of the crystallized nanoparticles sensibly increases from $\mathrm{S}_{21.5 \%}$ $(3.1 \%)$ to $\mathrm{S}_{26.6 \%}(4.8 \%)$.

The size dispersion and the optical constants variations with size influence simultaneously the calculated optical constants of the Si-NPs. While the influence of size dispersion is mainly perceptible on the broadening of $\varepsilon_{i}$ and its curvature near the absorption onset; the effect of the optical constants variations with size is noticeable on the transition energy but also on the broadening of $\varepsilon_{i}$. Particularly, our results put forward that the two aforementioned factors have opposite effects on the broadening of the imaginary dielectric function.

Moreover, Fig. 6 shows no significant increase in the amplitude of $\varepsilon_{i}$ derived from BEMA in the case of sample $\mathrm{S}_{21.5 \%}(\approx 34.8)$ to sample $\mathrm{S}_{26.6 \%}(\approx 34.4)$. Since the average size of the Si-NPS in $\mathrm{S}_{26.6 \%}$ is larger than in $\mathrm{S}_{21.5 \%}$, a meaningful increase in the amplitude would have been expected in that former composite layer. Table II suggests that, from sample $S_{21.5 \%}$ to sample $S_{26.6 \%}$, there is a more significant increase in the proportion of amorphous nanoparticles in comparison to the crystalline ones. Hence a third factor may also substantially influence the lineshape of the calculated optical constants: the relative proportions of the amorphous/ crystallized $\mathrm{Si}$-NPS.

It is very likely that a significant part of our $\mathrm{Si}-\mathrm{NPS}$ is ill-crystallized or amorphous (see Table II). At first sight, the optical characterization of such nanoparticles may not reveal any influence of size distribution on their optical properties owing to their amorphous character. Indeed the experimental highlight of quantum confinement effect (inter alia blueshift of the gap energy) in amorphous $\mathrm{Si}$ is not straightforward because the mean free path of the electron in $a-S i$ is rather short (of the order of $1 \mathrm{~nm}$ ). ${ }^{36}$ Nevertheless it has theoretically been demonstrated that quantum confinement is possible in amorphous Si nanostructures. ${ }^{37}$ Park et al. evidenced more recently, by using photoluminescence spectroscopy, the presence of quantum confinement in amorphous $\mathrm{Si}$ 
quantum dots within silicon nitride. ${ }^{38}$ Their results support the theoretical ones of Lannoo and coworkers. ${ }^{37}$ On that account the influence of size distribution on the physical properties of amorphous Si nanoparticles could be unveiled with the means of optical spectroscopy.

Our results put forward that not only the thermal budget (cf. comparison of the volume fractions in samples $\mathrm{S}_{16.8 \%}$ and $\mathrm{S}_{21.5 \%}$ in Table II) and the annealing type (cf. RTA or tube furnace) are significant factors affecting the crystallization process of the Si-NPs in silicon nitride; but also the initial quantity of $\mathrm{Si}$ introduced via the $\mathrm{SiH}_{4}$ precursor material is also determinant. Particularly, a comparison of the data concerning the Si-NPS in samples $\mathrm{S}_{10.2 \%} ; \mathrm{S}_{16.8 \%} ; \mathrm{S}_{26.6 \%}$; and $\mathrm{S}_{35.2 \%}$ suggests that a high silane flow (low $R_{Q}$ value) is needed in order to achieve a more efficient crystallization $\left(f_{c-S i}>10 \%\right.$ only in sample $S_{35.2 \%}$, according to Table II) of the nanoparticles in silicon nitride.

From the analysis presented here, one can point out a certain influence of the amorphous/crystalline character of the nanoparticles on the slope of their corresponding $\varepsilon_{i}$. A practical indicator may be given by the value of $\varepsilon_{\mathrm{i}}$ at $3.00 \mathrm{eV}$ obtained from BEMA: this value equals $12.5 ; 14.3 ; 15.9$; $21.3 ; 17.3 ; 16.5$; and 13.4 for samples $S_{10.2 \%}$ to $S_{35.2 \% \text {, }}$ respectively. A comparison of samples $\mathrm{S}_{10.2 \%}$ and

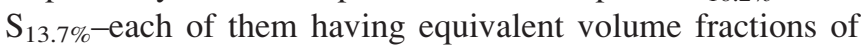
amorphous and crystallized $\mathrm{Si}-\mathrm{NPS}$-shows similar curvatures of $\varepsilon_{i}$ until almost $3.25 \mathrm{eV}$. Besides a comparison of specimens $\mathrm{S}_{16.8 \%} ; \mathrm{S}_{19.4 \%}$ and $\mathrm{S}_{26.6 \%}$-with equivalent fractions of crystallized Si-NPs and rising fractions of amorphous $S i-N P s$-indicates that the magnitude of $\varepsilon_{i}$ is higher for the nanoparticles with a dominant amorphous character. The observation of samples $S_{26.6 \%}$ and $S_{35.2 \%}$-with close fractions of amorphous Si-NPs and increasing fraction of crystallized $\mathrm{Si}-N P S$-suggests that crystallization of the nanoparticles induces a sensitive change in the curvature of $\varepsilon_{i}$. Our results indicate that amorphous Si-NPs have higher amplitude of $\varepsilon_{i}$ than the crystallized ones. This supports the observation of Gourbilleau et al. who noticed that absorption in $\mathrm{Si} / \mathrm{SiO}_{2}$ multilayered films is more important for amorphous Si quantum dots. ${ }^{39}$ It may appear difficult to disconnect the influence of the amorphous/crystalline character from that of size distribution on the lineshape of the dielectric function of the nanoparticles near their absorption onset. Nevertheless the results presented in Fig. 6 show the same tendency as those reported by Pesika et al. for $\mathrm{ZnO}$ quantum dots. ${ }^{13}$ Our results also support the idea that the absorption onset shows dependence with the size dispersion of the nanoparticles.

As expected, Table III shows that the values of $\sigma_{\min }$ and $\sigma_{\max }$ deduced appear to be consistent with the composition of the samples. Expectedly there is a relationship between $\sigma$ and $f_{S i-N P S}$ : the samples with the larger volume fractions of Si-NPs (e.g., sample $\mathrm{S}_{35.2 \%}$ ) have the larger size dispersions $\left(\sigma_{\min }\right.$ and $\sigma_{\max }$ equal to 1.30 and 1.35 , respectively, also for sample $\mathrm{S}_{35.2 \%}$ ). The variation ranges presented for $\sigma$ in Table III are coherent with the size dispersions previously reported for Si-NPs grown in either silica, ${ }^{3,10,30,31}$ or silicon nitride, ${ }^{40}$ or deposited on a surface. ${ }^{4}$ In these previous reports, the size dispersion of the Si-NPs were obtained experimentally by TEM and ranged between 1.15 and 1.35 .
The methodology introduced in this work enables the determination of the size dispersion $\sigma$ as well as the volume fraction $f_{S i-N P s}$ of the Si-NPs. This is done from ellipsometric measurement, without employing TEM, by comparing the lineshapes of $\varepsilon_{i}$ calculated from BEMA (used as the reference in this study) and $M M G$ models near the absorption onset of the nanoparticles.

By increasing the size dispersion $\sigma$, one would rather expect a redshift of the absorption peak as the contribution of the large-sized nanoparticles becomes more important. However, we observe a blueshift of the $\varepsilon_{\mathrm{i}}$ peak. Indeed in case of sample $\mathrm{S}_{26.6 \%}$, for example, the energy of the absorption peak moves from $3.30 \mathrm{eV}(\sigma=1.25)$ to $3.40 \mathrm{eV}$ $(\sigma=1.30)$. In our previous reports this observation led us to the assertion that as the size dispersion $\sigma$ rises, the transition energy $E$ and the optical gap $E^{04}$ of the Si-NPS increase, whereas the amplitude $A$ of the corresponding $\varepsilon_{i}$ decreases. ${ }^{23,24,33}$ It has to be clear in mind that these variations were stated only in the frame of the results of the numerical simulations. The latter were carried out starting from a unique effective dielectric function $\tilde{\varepsilon}_{\text {eff }}$. Consequently, the lineshape of the dielectric function computed for the ensemble of Si-NPs evolves in such a way that $\tilde{\varepsilon}_{\text {eff }}$ remains constant. One can notice that for each sample, the peaks of $\varepsilon_{i}$ derived from $M M G$ have a transition energy that gets closer to $B E M A$ than $M G$ with increasing value of $\sigma$. The observed blueshift of the energy peak (with increasing $\sigma$ ) in $M M G$ has to be interpreted as a tendency to reproduce the same lineshape of $\varepsilon_{\mathrm{i}}$ as $B E M A$. The reduction of the amplitude of $\varepsilon_{\mathrm{i}}$ noticed (mainly above $\approx 3.50 \mathrm{eV}$ ) with increasing $\sigma$ comes from the non-consideration of multipole interactions when the size of the nanoparticles gets larger. The corresponding lineshapes extracted from $M M G$ are necessary in order to derive information about the size dispersion of the nanoparticles. Such variations of $E^{04}\left(E_{g}\right)$ and $A$ versus $\sigma$, obtained when Eq. (5) of the $M M G$ model is used, are likely to be obtained for other composite systems similar to ours.

The evolutions observed (cf. Figs. 4 and 5) for the lineshape of $\varepsilon_{i}$ derived from $M G$ indicate the presence of aggregation in the investigated composite media. Apparently the aggregation threshold of the Si nanoparticles occurs at a volume fraction comprised between $10.2 \%$ and $13.7 \%$. In fact, we attribute such behavior to the microtopology of the distribution of the Si-NPS within the layer which seems to be more homogeneous with a close packing of the nanoparticles: samples $S_{26.6 \%}$ [cf. Fig. 1(b) and 1(d)] and $S_{35.2 \%}$ contain a more important proportion of larger nanoparticles as compared to the previous ones; therefore the response of these nanoparticles is likely to be dominant. Close values of the amplitude of $\varepsilon_{i}$ derived from $M G$ and BEMA may be a good indication that the sphere is a reasonable approximation for describing the shape of the nanoparticles. Particularly the annealing conditions performed (cf. Table I) explain the significant gaps observed for the values of $\varepsilon_{i}^{\max }$ computed by $M G$ and BEMA in case of samples $\mathrm{S}_{16.8 \%}, \mathrm{~S}_{19.4 \%}$ and $\mathrm{S}_{21.5 \%}$. The interparticle distance also affects the optical properties of the Si-NPs. ${ }^{16,26,41}$ This is evidenced in the evolution of the peak position $E^{\max }$ and explains the non-realistic 
resonance energies derived from $M G$ for $f_{S i-N P s}>13.7 \%$ in our systems.

The values of $\sigma_{\min }$ and $\sigma_{\max }$ are in fair agreement with TEM histograms. Indeed for sample $\mathrm{S}_{26.6 \%}$, we show in Table III that the size distribution of nanoparticles varies between 1.25 and 1.30. This observation agrees with the TEM image (see Fig. 2(b)). In addition, in the case of sample $S_{19.4 \%}$, the standard deviation $\sigma$ varies between 1.15 and 1.20. This value is also in agreement with TEM image [see Fig. 1(a)] and seems reasonable in regard of the small size of nanoparticles. According to Table III, there is a sensitive dependence of the bounds $\left[\sigma_{\min }-\sigma_{\max }\right]$ with the volume fraction (hence the mean size) of the Si-NPs and in fine with the annealing conditions of the samples. Several reasons can be put forward in order to explain the observed gaps between TEM and the values of $\sigma$ determined by surrounding $\varepsilon_{i}$ of $B E M A$ by the $\varepsilon_{i}$ of $M M G$. First, the differences may come from the values employed for $R_{\min }$ and $R_{\max }$ in the integrand of Eq. (6). Small deviations of $R_{\min }$ and $R_{\max }$ from the true values could lead to substantial variations of $\sigma$ derived from $M M G$ as compared to TEM. Second, the surface and volume of the characterized regions change significantly from TEM to ellipsometry, thus leading to different sampling of the semiconductor nanoparticles.

Even though it is well known that the accuracy and precision of ellipsometry is considerably reduced as $k \rightarrow 0$, the present estimations of $\sigma$ are, in our opinion, trustworthy since the lower and upper limits of $I_{E}$ always are located between $2.00 \mathrm{eV}$ and $3.00 \mathrm{eV}$. At such photon energy, the value of the extinction coefficient is significant and on that account can be precisely measured. ${ }^{25}$

We have pointed out before uncertain evolutions of the dielectric function of Si nanocrystals embedded in silica. ${ }^{23,24}$ Indeed conflicting lineshapes were presented for $\varepsilon_{i}$ whereas the size distribution of the $\mathrm{Si}$ nanocrystals differed only slightly. The authors of Ref. 31 found that for Si nanocrystals of $4.2 \mathrm{~nm}$ in diameter (with an estimated $\sigma$ close to $\approx 1.20$ according to their corresponding TEM histograms), the absorption onset of $\varepsilon_{i}$ starts only above $3 \mathrm{eV}$. This behavior is in inconsistency with the lineshape of $\varepsilon_{i}$ derived by other groups who also characterized Si nanocrystals in silica with close mean diameters and size dispersion. ${ }^{27,30}$ We suggest here that a consideration of the size distribution in the derivation of the optical properties of Si nanocrystals by spectroscopic ellipsometry may certainly clear up the striking discrepancies noticed in the investigations presented in the literature.

It can be noticed that the figures of $n_{\text {eff }}$ (see Table IV) deduced from $B E M A$ are always surrounded by two distinct values calculated from $M M G$. The estimated values of the size dispersion $\sigma$ of the nanoparticles are in good agreement with the previous one presented in Fig. 6 and Table III. This result supports the underlying idea of Eq. (5) displayed in Fig. 2. Evidently it may be possible to derive more precise values for $\sigma$ corresponding to each investigated $S i$-NPs but such task is out of the scope of this paper. The results presented here convey the idea that the consideration of size distribution enables to set up bounds on the dielectric function of the ensemble of Si nanoparticles. In analogy with the relationship (6), the dielectric function of an ensemble of Si-NPs should strictly evolve close to a specific lineshape that is determined by the values of $f_{S i-N P S}$ and $\sigma$ : obtaining lineshapes for $\varepsilon_{\mathrm{i}}$ of the Si nanoparticles varying outside these specific bounds, near the absorption onset (id est for photon energy range up to $\approx 3 \mathrm{eV}$ ), should then be arguable in respect with the modeling procedure employed for the derivation of $\varepsilon_{i}$ and its physical meaning [see Figure 3 of Ref. 23]. Hence, the $M M G$ model introduced in this work can help finding the accurate evolution for the lineshape of $\varepsilon_{i}$ (and $\varepsilon_{r}$ ) for an ensemble of Si nanoparticles by taking into account their size dispersion $\sigma$.

It is noteworthy that from two radically different effective medium models (modified $M G$ and BEMA), very similar lineshapes for the dielectric function of $\mathrm{Si}$ nanoparticles have been successfully obtained up to approximately $3 \mathrm{eV}$, by extracting critical information about the size dispersion of the nanoparticles, and thus confirming the realistic nature of the dielectric functions obtained from $B E M A$. The latter model has been used as a reference in the present study, since it provides consistent results from a physical point of view. Besides, the reasonable values of $\sigma$ deduced from the method presented here imply that the effective dielectric functions $\tilde{\varepsilon}_{\text {eff }}$ that have been initially derived [by optical model of Fig. 3(b)] are likely to correspond to the accurate (most realistic) ones for the investigated nanocomposite systems. The present results finally convey the idea that the use of a bulk-like dielectric function $\tilde{\varepsilon}(\mathrm{E})$ which is not explicitly dependent on the size $R$ of the nanoparticles [cf. Eq. (5)] remains somehow valid, at least in the case of systems comparable to ours.

\section{CONCLUSION}

In this paper, the dielectric responses of $\mathrm{Si}$ nanoparticles in a nitride matrix have been determined by Bruggeman effective medium approximation; Maxwell-Garnett model and a modified Maxwell-Garnett model that allows the explicit consideration of size dispersion $\sigma$ of the nanoparticles. The ellipsometric data were analyzed through two different optical models which either take explicitly into account the composite structure of the film or consider it as a homogeneous layer. For the investigated systems, BEMA remains the most accurate model in comparison to $M M G$ and $M G$. The Maxwell-Garnett model fails in describing properly the optical properties of the Si nanoparticles above a volume fraction of $10.2 \%$. The discrepancy between the BEMA and $M G$ models increases with the volume fraction of the nanoparticles. Besides, the knowledge of $\sigma$ is absent in $B E M A$ and $M G$. We have demonstrated that, within a restricted energy range close to the absorption onset of the nanoparticles, the dielectric functions calculated from a size-distribution dependent extension of Maxwell-Garnett formula, with two specific values of $\sigma$, surround the dielectric function calculated from Bruggeman model. The values thus derived for $\sigma$ are in acceptable agreement with size dispersions obtained from energy filtered electron microscopy histograms. Moreover the calculated effective number of 
electrons derived enables concluding that the parameter $\sigma$ represents the size dispersion. In this way, we have provided a self-consistent methodology for the computation of the dielectric function of Si nanoparticles from an ellipsometric measurement. The consideration of $\sigma$ in the modeling procedure of Si nanoparticles brings additional information about the microtopology of the composite medium. It may thus be possible to use spectroscopic ellipsometry for the accurate derivation of the dielectric function of silicon nanoparticles and estimation (in situ control) of their size dispersion $\sigma$ at the same time. Extension to other systems of semiconductor nanoparticles within a dielectric matrix can therefore be considered using the present methodology.

\section{ACKNOWLEDGMENTS}

A.S.K. and A.E.N. acknowledge Horiba Jobin Yvon, Inc. for enabling ellipsometric measurements over an extended spectral range.

${ }^{1}$ S. Tiwari, F. Rana, H. Hanafi, A. Hartstein, E. F. Crabbé, and K. Chan, Appl. Phys. Lett. 68, 1377 (1996).

${ }^{2}$ J. Heitmann, F. Müller, M. Zacharias, and U. Gösele, Adv. Mater. 17, 795 (2005).

${ }^{3}$ F. Iacona, G. Franzò, and C. Spinella, J. Appl. Phys. 87, 1295 (2000).

${ }^{4}$ C. Meier, A. Gondorf, S. Lüttjohann, A. Lorke, and H. Wiggers. J. Appl. Phys. 101, 103112 (2007).

${ }^{5}$ S. Schamm, C. Bonafos, H. Coffin, N. Cherkashin, M. Carrada, G. Ben Assayag, A. Claverie, M. Tencé, and C. Colliex. Ultramicroscopy 108, 346 (2008)

${ }^{6}$ W. Grogger, B. Schaffer, K. M. Krishnan, and F. Hofer, Ultramicroscopy 96, 481 (2003).

${ }^{7}$ C. R. M. Oliveira, A. M. de Paula, F. O. Plentz Filho, J. A. M. Neto, L. C. Barbosa, O. L. Alves, E. A. Menezes, J. M. M. Rios, H. L. Fragnito, C. H. B. Cruz, and C. L. Cesar, Appl. Phys. Lett. 66, 439 (1995).

${ }^{8}$ M. Hirasawa, T. Oni, and T. Seto, Appl. Phys. Lett. 88, 093119 (2006).

${ }^{9}$ A. G. Rolo and M. I. Vasilevskiy, J. Raman Spectrosc. 38, 618 (2007).

${ }^{10}$ L. Mercaldo, P. D. Veneri, I. Usatii, E. M. Esposito, and G. Nicotra, Sol. Energy Mater. Sol. Cells 119, 67 (2013).

${ }^{11}$ T. Arguirov, T. Mchedlidze, M. Kittler, R. Rölver, B. Berghoff, M. Först, and B. Spangenberg, Appl. Phys. Lett. 89, 053111 (2006).

${ }^{12} \mathrm{P}$. Y. Yu and M. Cardona, Fundamentals of Semiconductors, Physics and Materials Properties, 4th ed. (Springer-Verlag, Berlin, 2010), Chaps. 3 and 7.

${ }^{13}$ N. S. Pesika, K. J. Stebe, and P. C. Searson, J. Phys. Chem. B 107, 10412 (2003).

${ }^{14}$ D. Bergman, Phys. Rep. 43, 377 (1978).
${ }^{15}$ W. Theiß, Surf. Sci. Rep. 29, 91 (1997).

${ }^{16} \mathrm{~J}$. Humlicek, "Data analysis for nanomaterials: Effective medium approximation, its limits and implementations," in Ellipsometry at the Nanoscale, edited by M. Losurdo and K. Hingerl (Springer-Verlag, Berlin, 2013).

${ }^{17}$ L. Bányai and S. W. Koch, Semiconductor Quantum Dots (World Scientific, Singapore, 1993), Chap. 2, pp. 20-29.

${ }^{18}$ D. E. Aspnes, Thin Solid Films 89, 249 (1982).

${ }^{19}$ A. Spanoudaki and R. Pelster, Phys. Rev. B 64, 064205 (2001).

${ }^{20}$ R. G. Barrera, P. Villaseñor-González, W. L. Mochán, and G. Monsivais, Phys. Rev. B 41, 7370 (1990).

${ }^{21}$ L. G. Grechko, V. N. Pustovit, and K. W. Whites, Appl. Phys. Lett. 76, 1854 (2000).

${ }^{22}$ P. Chýlek and V. Srivastava, Phys. Rev. B 27, 5098 (1983).

${ }^{23}$ A.-S. Keita and A. En Naciri, Phys. Rev. B 84, 125436 (2011).

${ }^{24}$ A.-S. Keita, A. En Naciri, F. Delachat, M. Carrada, G. Ferblantier, and A. Slaoui, Appl. Phys. Lett. 99, 131903 (2011).

${ }^{25}$ Handbook of Ellipsometry, edited by H. G. Tompkins and E. A. Irene (William Andrew, Norwich, NY, 2005), Chaps. 2 and 3.

${ }^{26}$ D. E. Aspnes, Am. J. Phys. 50, 704 (1982).

${ }^{27}$ L. Ding, T. P. Chen, Y. Liu, C. Y. Ng, and S. Fung, Phys. Rev. B 72, 125419 (2005).

${ }^{28}$ H.-C. Weissker, J. Furthmüller, and F. Bechstedt, Phys. Rev. B 67, 165322 (2003).

${ }^{29}$ Y. Battie, A. Resano-Garcia, N. Chaoui, N. Zhang, and A. E. Naciri, J. Chem. Phys. 140, 044705 (2014).

${ }^{30}$ B. Gallas, I. Stenger, C.-C. Kao, S. Fisson, G. Vuye, and J. Rivory, Phys. Rev. B 72, 155319 (2005)

${ }^{31}$ M. I. Alonso, I. C. Marcus, M. Garriga, A. R. Goñi, J. Jedrzéjewski, and I. Balberg, Phys. Rev. B 82, 045302 (2010).

${ }^{32}$ R. Espiau de Lamaëstre and H. Bernas, Phys. Rev. B 73, 125317 (2006).

${ }^{33}$ A. En Naciri, P. Miska, A.-S. Keita, Y. Battie, H. Rinnert, and M. Vergnat, J. Nanopart. Res. 15, 1 (2013).

${ }^{34}$ A.-S. Keita, A. En Naciri, F. Delachat, M. Carrada, G. Ferblantier, and A. Slaoui, J. Appl. Phys. 107, 093516 (2010).

${ }^{35}$ M. Carrada, A. Zerga, M. Amann, J. J. Grob, J. P. Stoquert, A. Slaoui, C. Bonafos, and S. Schamm, Mater. Sci. Eng. B 147, 218 (2008).

${ }^{36}$ M. Beaudoin, M. Meunier, and C. J. Arsenault, Phys. Rev. B 47, 2197 (1993).

${ }^{37}$ G. Allan, C. Delerue, and M. Lannoo, Phys. Rev. Lett. 78, 3161 (1997).

${ }^{38}$ N.-M. Park, C.-J. Choi, T.-Y. Seong, and S.-J. Park, Phys. Rev. Lett. 86, 1355 (2001).

${ }^{39}$ F. Gourbilleau, C. Ternon, D. Maestre, O. Palais, and C. Dufour, J. Appl. Phys. 106, 013501 (2009).

${ }^{40}$ F. Delachat, M. Carrada, G. Ferblantier, J.-J. Grob, and A. Slaoui, Nanotechnology 20, 415608 (2009).

${ }^{41}$ K. Seino, F. Bechstedt, and P. Kroll, Mater. Sci. Eng. B 177, 1098 (2012). 